\section{Military Technical College, Kobry El-Kobbah,} Cairo, Egypt

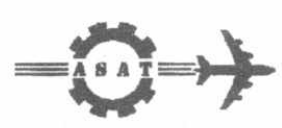

$9^{\text {th }}$ International Conference

On Aerospace Sciences \& Aviation Technology

\title{
MODELING OF SHAPED CHARGE JET FORMATION
}

By

Mohamed, S.Y. , Riad, A.M. and Kresha Y."

\section{ABSTRACT}

The PER theory that developed to describe the collapse process of a conical shaped charge liner has been modified. New algorithms are used to determine the deflection angle, the collapse angle and the collapse velocity of each collapsed liner element. In the modified model, the liner is divided into $n$ elements. For each element of collapsed liner, the main equations determining deflection angle, collapse angle, collapse velocity, jet velocity and jet mass are derived. These equations are arranged and compiled into a computer program. The input data to the program are easily determined

The model is capable of predicting the changes of collapse velocity, jet velocity, and deflection and collapse angles, respectively, with relative axial position of liner element. The model is capable also of predicting the total mass of the formed jet. The predictions of the model are compared with numerical and experimental results of other investigators; good agreement is obtained. Moreover, representative samples of the model predictive results using small sizes and the $105 \mathrm{~mm}$ shaped charges, respectively, are presented and discussed

\section{KEY WORDS}

Explosive loading, Shaped charge, Explosive-metal interaction, and Jet formation

\footnotetext{
* Egyptian Armed Forces.

\# Assoc. Prof., Head of R. \& D. Dept., Helwan Industrial Eng. Company. Cairo, Egypt.
} 


\section{INTRODUCTION}

The importance of lined shaped charges in both civil and military applications attracts the attention of many investigators to model the shaped charge phenomena. The jet formation process represents the initial phase of a such phenomena. The good predictions of shaped charge performance are essentially based on the well established simulation of the jet formation process

Jet formation starts after explosive detonation. Upon detonation, a spherical detonation wave propagates outward from the point of detonation. This high pressure wave moves at a very high velocity, typically around $8 \mathrm{~km} / \mathrm{s}$. As the detonation wave strikes the liner, the material is accelerated under the high detonation pressure, collapsing the cone. During this process, shown in Fig.1, for a typical conical liner, the liner material is driven to very high distortions over very short time intervals. The collapse of the conical liner material on the centerline forces a portion of the collapsed liner to flow in the form of a jet where the jet tip velocity can travel in excess of $10 \mathrm{~km} / \mathrm{s}$. Because of the presence of a velocity gradient, the jet will stretch until it fractures into a column of "jagged" particles [1]

Two main theories were developed to describe the jet formtion process; these were: (i) steady state, and (ii) unsteady state theories. Birkhoff et al. [2] developed the steady state theory of a conical shaped charge jet formation. They assumed that: (a) the detonation wave produced a very high pressure during the collapse process, (b) the pressure at the wave front was higher than the strength of the liner material, (c) the liner material was treated as an inviscid, incompressible fluid, and (d) the collapsed liner elements were instantenously accelerated to their final collapse velocities. The theory predicted that the formed jet length was constant and was equal to the length of liner wall.

Pugh et al. [3] had modified the steady state theory of Birkhoff et al.; their theory was named "unsteady state" or "PER" theory. They considered that the collapse velocities of the liner elements were not equal but decreased continuously from the cone apex to its base, producing significant jet elonagtion. The concept of variable collapse velocities of the liner elements allowed the PER theory to predict better results of jet formation process than the steady state theory. A geometrical scheme of the liner collapse process is shown in Fig. 2 .

Chou et al. [4] derived an expression determining the deflection angle of each collapsed element of liner $\delta$ when the time history of the collapse velocity was available. It was mentioned by Jones [5] and Chanteret [6] that the expression of Chou et al. gave better predicted values for the deflection angle when they compared with the corresponding experimental results. This expression is also recommended to calculate the deflection angle by Liu [7].

The collapse angle $\beta$ determined by PER theory was greater than that of the steady state angle $\beta^{+}$, cf. Fig. 2. It was assumed by PER theory that each liner element was thin and not affected by its neighbours. Moreover, the collapse angle was a function of deflection angle, half of cone angle, liner element position, collapse velocity and its partial derivatives with respect to liner element position. Different methods were used for calculating the collapse angle; these were: (i) expressing the collapse angle as the sum of the steady state angle $\beta^{+}$and the angle $\Delta \beta[4]$, and (ii) using a pseudo-collapse angle $\beta^{-}$instead of $\beta$ [6]. These methods gave better results than those of the original PER theory.

The PER theory assumed that the collapse velocity of each liner element was instantenously increased to the axis as shown in Fig. 3a. Carleone et al. [see Ref. [8]] proposed that the velocity was increased linearly over a short period until it reached its 
final value on the axis, cf. Fig. 3b. Randers [see Ref.[1] proposed a more realistic form of the collaspe velocity-time history; it was an exponetial function as shown in Fig. 3c. The constant $\tau$ of the function was determined empirically by Carleone and Chou [see Ref.[8]]. whereas Jones [5] determined the value of this constant by iteration.

In this paper, the original PER theory has been modified. Alternative algorithms are used to determine the deflection angle, the collapse angle and the collapse velocity of each collapsed liner element. The main equations representing the modified theory have been presented. These equations are arranged and compiled into a computer program. The results of the modified theory are validated with numerical and experimental results of other investigators. Moreover, predicted samples using small sizes and the $105 \mathrm{~mm}$ shaped charges, respectively, are presented and discussed.

\section{ANALYTICAL MODEL}

In this model, the liner is assumed to be divided into $n$ elements. As the detonation wave front arrives the liner element at the point $P$, it starts to collapse inward to the charge axis with a velocity $V_{0}$; cf. Fig. 2 . This velocity makes an angle with the normal to the liner surface named the deflection angle $\delta$. Moreover, the collapse angle $\beta$ of the liner element is greater than the half of cone angle $\alpha$. When the detonation wave front travels from the point $P$ to the point $Q$ along the surface $A P Q$, the liner element at $P$ collapses to $J$. The liner element at the point $\mathrm{P}^{\prime}$ starts later to collapse with a velocity lower than that at the point $P$ and arrives to the point $M$ at the same time as $P$ arrives $\mathrm{J}$. Because the collapse velocities of the liner elements are not identical, the collapsed element at P' doesn't reach $\mathrm{N}$ as $\mathrm{P}$ arrives to $\mathrm{J}$ and the collapsing liner has a curved contour $\mathrm{QMJ}$ as shown in Fig. 2

\subsection{Modeling the Deflection Angle $\delta$}

For each collapsed liner element, Taylor [see Ref. [5]] developed a formula determining the deflection angle $\delta$ as a function of its collpase velocity $V_{0}$, the detonation velocity $U_{D}$ and the half of the cone apex angle $\alpha$. This formula is:

$$
\delta=\sin ^{-1}\left(V_{0} \cos \alpha / 2 U_{0}\right) \text {. }
$$

To use Eqn. (1), the collpase velocity of each liner element must be available. A lot of formulae determining the velocity imparted to a metal in contact with a detonating explosive and often used to determine the collpase velocity of shaped charge liner were developed $[1,7,9]$. The following equation is selected, based on its good predictions when compared with experimental measurements, to predict the collapse velocity $V_{0}$ of each liner element; this equation is [1]:

$$
V_{0}=1.2 U_{D}\left[\frac{\sqrt{1+32 /(27 \mu)}+1}{\sqrt{1+32 /(27 \mu)}-1}\right] \text {, }
$$

where $\mu$ is the ratio of the mass of the liner element to the mass of the explosive above this element. The ratio $\mu$ is determined using the following equation; cf. Fig. 4:

$$
\begin{aligned}
\mu & =M_{L i} / M_{\text {expi }} \\
& =\left(2 T_{L} \tan \alpha X_{i} \rho_{L}\right) /\left(\left[0.25 D_{B}^{2}-X_{i}^{2} \tan ^{2} \alpha\right] \rho_{\exp }\right),
\end{aligned}
$$


where $M_{L i}$ is the mass of the collasped element of the liner, $M_{\text {expi }}$ is the mass of explosive above this element, $T_{L}$ is the thickness of liner wall, $X_{i}$ is the axial position of liner element, $D_{B}$ is the base diameter of the cone, $\rho_{L}$ is the density of liner material, and $\rho_{\exp }$ is the denisty of explosive material.

The collapsed liner elements near the apex haven't the sufficient times to attain their final collapse velocities. As the distance between the liner element and the apex of cone increases, more time is available for the collapsed element to be accelerated. Because of the decrease of explosive masses above the farest elements of liner, these elements haven't also attain their final collapse velocities. Therefore, the determination of the collapse velocity of each liner element using Eqn. (2) doesn't represent the real value

To determine the real collapse velocity of each liner element, the developed equation by Randers [see Ref.[4]] that determines the time history of the collapse velocity of each element is used. This equation is represented by:

$$
V(t)=V_{0}\left(1-e^{-\frac{t-t_{0}}{\tau}}\right)
$$

where $t$ is the time taken by the collapsed element of liner to reach the charge axis, $t_{0}$ is the time taken by the detonation wave front to reach the liner element, and $\tau$ is the time constant. The value of the constant $\tau$ is determined using the following equation [10]:

$$
\tau=m_{\mathrm{Li}} V_{0} / P_{\mathrm{cj}_{\mathrm{j}}},
$$

where $m_{\mathrm{Li}}$ is the mass of the liner element per its lateral area and $P_{\mathrm{Cj}}$ is the ChapmanJougouet pressure. The term $m_{L}$ is calculated using the following equation; cf. Fig. 4 :

$$
\begin{aligned}
m_{L 1} & =M_{L i} / A_{L} \\
& =\left(2 R_{i} T_{L} \rho_{L}\right) /\left(\tan \alpha\left[2 X_{1}-(L / n)\right]\right),
\end{aligned}
$$

where $A_{L}$ is the lateral area of the liner element, $R_{i}$ is the radius of the liner element, $L$ is the height of the cone, and $\mathrm{n}$ is the total number of liner elements.

The equation representing the Chapman-Jougouet pressure is [6]

$$
P_{c}=0.25 \rho_{\text {expo }} U_{c}^{2} \text {. }
$$

For each liner element, the real value of its coliapse velocity is determined using an iterative method suggested by Jones [5] and successfully used by Liu [7] as follows:

a) Using the geometry of the conical liner, the distance of the collapsed liner element from the axis of symmetry, named DIST, is determined by:

$$
\text { DIST }=X_{1} \tan \alpha / \cos (\alpha+\bar{\delta}) \text {. }
$$

The deflection angle $\delta$ is calculated using Eqn.(1)

b) The distance of the collapsed liner element from the axis of symmetry is re-calculated as the integration of its collapse velocity with respect to time, cf. Eqn (4). This distance is named DI and represented by 


$$
\begin{aligned}
D I & =\int_{t_{0}}^{t} V(t) d t \\
& =V_{0}^{*}\left(t-t_{0}\right)+\tau^{*} V_{0}^{*}\left(e^{\frac{t_{0}-t}{t}}-1\right) .
\end{aligned}
$$

c) Substituting into Eqn. (9) using different values of time t. The iterative process ends when the value of DI is very close to the value of DIST determined by Eqn. (8); the corresponding time $t$ is denoted $t_{f}$.

d) Substituting into Eqn. (4) by the value of the time $t_{f}$, the calculated velocity represents the real collapse velocity of liner element and is denoted by $\mathrm{V}_{\text {real. }}$.

Once the real collapse velocity of each collapsed liner element is determined, the deflection angle $\delta$ is re-calculated by replacing the collapse velocity $V_{0}$ in Eqn. (1) by the corresponding real collapse velocity $V_{\text {real }}$. The final deflection angle $\delta$ of each collapsed liner element is determined by:

$$
\delta=\sin ^{-1}\left(V_{\text {real }} \cos \alpha / 2 U_{D}\right) .
$$

\subsection{Modeling the Collapse Angle $\beta$}

Many investigators have modeled the difference between the collapse angle of the steady state theory $\beta^{+}$and the corresponding angle of the unsteady state theory $\beta$, cf. Fig. 2. In the present model, the collapse angle $\beta$ associated with each collapsed liner element is determined using Hirsch's equation [see Ref. [7]]. This equation is:

and

$$
\begin{aligned}
& \beta=\left(\beta^{+}+\Delta \beta\right), \\
& \beta^{+}=\alpha+2 \delta .
\end{aligned}
$$

The deflection angle $\delta$ is calculated using Eqn. (10) whereas, the angle $\Delta \beta$ is determined using the following equation [see Ref. [7]]:

$$
\Delta \beta=\tan ^{-1}-\left(X_{i} \sin \alpha /[\cos (\alpha+\delta) \cos \delta]\right)\left(V_{\text {reaa }}^{\prime} / N_{\text {real }}\right),
$$

where $V_{\text {real }}$ is the real collapse velocity of each liner element and $V_{\text {real }}$ is the partial derivative of the real collapse velocity with respect to the distance of the liner element from the liner apex $X_{i}$.

The real collapse velocity is represented as a function of the time at which each collapsed liner element reaches the charge axis:

$$
V_{\text {real }}=V_{0}\left(1-e^{-t^{t}-t_{0}} \tau^{-}\right)
$$

Using Eqns. (2) and (13), the partial derivative of $V_{\text {real }}$ with respect to the distance of the liner element from the liner apex $X_{i}$ is determined using the following equation:

$$
V_{\text {real }}=\left(d V_{\text {rea }} / d X_{i}\right)=\left(d V_{0} / d \mu\right)^{*}\left(d \mu / d X_{i}\right)^{*}\left(1-e^{\frac{t}{t-t_{0}} \tau}\right)
$$


Once $\Delta \beta$ is determined using Eqn. (12), the collapse angle $\beta$ is calculated for each collapsed liner element using Eqn. (11)

\subsection{Modeling the Jet Velocity and its Mass.}

The geometric relationships at the moving junction $\mathrm{R}$ are shown in Fig. 5. The axis of the cone is along RJ. PR is the element of liner moving toward the axis and has a velocity $V$ in moving coordinates. The velocity of the moving coordinates is $R J=V_{1}$. Using the law of sines from Fig. 5 yields [1]:

and

$$
\mathrm{V}=\mathrm{V}_{\text {real }} \cos (\alpha+\delta) / \sin \beta \text {, }
$$

$$
V_{1}=V_{\text {real }} \cos (\beta-\alpha-\delta) / \sin \beta
$$

In fixed coordinates, the jet velocity of each liner element is determined by [1]

$$
V_{1}=V+V_{1}
$$

Substituting by Eqns. (15)a and (15)b into Eqn. (16), the jet velocity of each liner element is represented by:

$$
V_{j}=V_{\text {real }}{ }^{*} \operatorname{cosec}(\beta / 2)^{*} \cos (\alpha+\delta-(\beta / 2)) \text {. }
$$

For determining the jet velocity of each liner element using Eqn. (17), the values of the deflection angle $\delta$, the collapse angle $\beta$ and the real collapse velocity $V_{\text {real }}$ associated with each collapsed element of liner are calculated using Eqns. (10), (11) and (13), respectively

Let $\mathrm{M}_{\mathrm{L}}$ be the mass of collapsed element of liner, $\mathrm{m}_{\mathrm{j}}$ be the part of mass of collapsed liner element entering the jet whereas, $m_{s i}$ be the mass of liner element entering the slug Applying the mass conservation principle results in:

$$
M_{\mathrm{LI}}=m_{\mathrm{H}}+\mathrm{m}_{\mathrm{S}} \text {. }
$$

Equating the horizontal momentum components of masses entering and leaving the junction R, cf. Fig. 5, yields:

$$
M_{\mathrm{Li}} V \cos \beta=m_{\mathrm{si}} V-m_{\mathrm{ji}} V
$$

Combining Eqns. (18) and (19) results in:

$$
m_{\mathrm{j} \mid}=M_{\mathrm{L}}{ }^{*} \sin ^{2}(\beta / 2) \text {. }
$$

The total jet mass represents the sum of the masses of liner elements entering the jet

The main equations representing the analytical model of jet formation process are derived These equations are arranged and compiled into a computer program. The input data to the program are easily determined. These data are the geometrical dimensions of the liner, and the densities of both liner and explosive materials.

\section{RESULTS AND DISCUSSIONS}

The relative axial position of liner element, axial position of each element $X_{\text {, divided by the }}$ liner height $\mathrm{L}$, is considered as an independent parameter. For each liner element, the 
present model is capable of predicting the change of deflection angle, collapse angle, collapse and jet velocities, and jet mass as a function of relative axial position of liner element. In addition, the model is also capable of predicting the total mass of the formed jet. In the following, the predicted results of the model are compared with experimental and numerical results of other investigators. Moreover, representative samples of model predictions using small sizes and the $105 \mathrm{~mm}$ shaped charges are presented and discussed.

\subsection{Validation of Model Results}

Allison and Vitali [see Ref. [1]] determined experimentally the change of jet velocity with relative axial position of liner element for the $105 \mathrm{~mm}$-conical shaped charge. The tested shaped charge has an apex angle of $42^{\circ}$ and a copper liner of thickness $2.69-\mathrm{mm}$. In addition, they used an explosive charge which has a plane wave detonation velocity of 8 $\mathrm{Km} / \mathrm{s}$. The measured jet velocity of the tested shaped charge starts when the relative axial position of the liner element is equal to 0.4 . Walters and Zukas [1] used a numeric code named BASC to predict the change of jet velocity with relative axial position of liner element for the same shaped charge.

The present model is run using the data of the shaped charge tested by Allison and Vitali. Figure 6 plots the predicted change of jet velocity with relative axial position of liner element. In addition, the predicted change of jet velocity with relative axial position of liner element obtained by Walter and Zukas and the corresponding experimental measurements obtained by Allison and Vitalli, respectively, are depicted on the same figure. Good agreement between the predicted results of the present model, numerical results of Walters and Zukas and experimental measurements of Allison and Vitalli is obtained.

Another validation of the jet formation modeling is performed. The model is tested using the same data of the $100 \mathrm{~mm}$-shaped charge that was tested by Chanteret [6]. The tested shaped charge has a cone apex angle of $60^{\circ}$ and a copper liner thickness of $2 \mathrm{~mm}$. The used explosive charge consists of a mixture of Hexogen and Tri-nitrotoluene with equal percent; the explosive charge is provided with a wave shaper.

Figure 7 plots the predicted change of jet velocity with relative axial position of liner element for the $100 \mathrm{~mm}$ shaped charge. The corresponding experimental measurements obtained by Chanteret [6] are depicted on the same figure. The predicted results of the present model are in good agreement with the experimental measurements of Chanteret except for the region where $X_{i} / L \geq 0.85$. This may be attributed to the effect of wave shaper. The shaper increases the velocities of the jet elements originated from the region near to the liner base at which a small amount of explosive is used to collapse them.

A comparison of the model predictions with the numerical results of Walter and Zukas [1] is done. They used the BASC code to predict the change of jet velocity with relative axial position of liner element, cf. Fig. 8. They fed the code with the data of a shaped charge; liner and explosive charge. The copper liner of the charge has a base diameter of 81.3 $\mathrm{mm}$, an apex angle of $42^{\circ}$ and a wall thickness of $1.9 \mathrm{~mm}$. Octal has a density of 1.717 $\mathrm{g} / \mathrm{cm}^{3}$, a detonation velocity of $7890 \mathrm{~m} / \mathrm{s}$ and a gurney velocity of $2821 \mathrm{~m} / \mathrm{s}$. These data are also fed into the program representing the present model and the change of jet velocity with relative axial position of liner element is predicted and plotted on the same figure. The predicted jet velocity of the present model is in a good agreement with the corresponding numerical ones of the BASC code. Moreover, the maximum difference between the predicted and numerical jet velocity is $11 \%$ at relative axial position of 0.3 . 


\subsection{Current Prediced Results}

In the following, representative samples of the model predictions using small sizes shaped charges, designated by $\mathrm{Ch} 1$ and $\mathrm{Ch} 2$, and the $105 \mathrm{~mm}$ shaped charge, respectively, are presented [11]. The input data to the program of the $\mathrm{Ch} 1, \mathrm{Ch} 2$ and the $105 \mathrm{~mm}$ charges are listed in Table 1. The predictions of the main parameters associated with the jet formation process are determined as a function of relative axial position of the liner element.

Figure 9 plots the predicted change of collapse velocity with relative axial position of liner element for the charges $\mathrm{Ch} 1, \mathrm{Ch} 2$ and the $105 \mathrm{~mm}$, respectively. For each shaped charge, it is noticed that the collapse velocity starts to rise from an initial value to its maximum. This increase is due to the so-called inverse velocity gradient where the collapsed liner elements near to the cone apex do not have sufficient times to attain their final flow velocities before arriving the cone axis. With the increase of relative axial position of liner element, more space (time) is available for each collapsed liner element to be accelerated. The maximum real collapse velocity represents the point at which the collapsed liner element has a sufficient time to attain its highest flow velocity before arriving the cone axis.

For the subsequent liner elements, the increase of the ratios of their masses to the explosive masses above these elements decreases their collapse velocities. Therefore, the change of collapse velocity for these elements of liner begins to decrease with the increase of relative axial position. The model predicts the maximum collapse velocities of the liner for the charges Ch1, Ch2 and the $105 \mathrm{~mm}$, respectively; these velocities are 2915,3205 and $3518 \mathrm{~m} / \mathrm{s}$.

Figure 10 plots the predicted change of deflection angle with relative axial position of liner element for the same charges. For each shaped charge, because of the deflection angle of each collapsed element of liner is a function of its collapse velocity, the trend of the change of deflection angle with relative axial position is similar to that of the collapse velocity. The deflection angle increases to its maximum value then it decreases to a minimum one. The model predicts that the maximum deflection angles for the charges Ch1, Ch2, and $105 \mathrm{~mm}$ are $30^{\circ}, 17.5^{\circ}$ and $13.75^{\circ}$, respectively; these angles are obtained at relative axial positions of $0.25,0.25$ and 0.35 whereas, the predicted minimum deflection angles are $6^{\circ}, 2.25^{\circ}$ and $5^{\circ}$, respectively, at relative axial positions of 1 .

Figure 11 plots the predicted change of collapse angle with relative axial position of liner element for the same charges. For each shaped charge, it is clear from the figure that the collapse angle is continuously increased with relative axial position of liner element. The model predicts that the maximum collapse angles for $\mathrm{Ch} 1, \mathrm{Ch} 2$ and the $105 \mathrm{~mm}$ are $118^{\circ}$, $111^{\circ}$ and $82^{\circ}$, respectively. These maximum angles are corresponding to relative axial positions of 1 .

Figure 12 plots the predicted change of jet velocity with relative axial position of liner elements for the charges ch1, ch2 and the $105 \mathrm{~mm}$, respectively. Because the jet velocity of each liner element is a function of its collapse velocity, the trend of the change of jet velocity with realtive axial position is similar to that of the collapse velocity, cf. Fig. 9 . For each shaped charge, the jet velocity increases until it reaches its maximum value, assuming that it represents the tip velocity of the jet, then it continuously decreases. The decreasing rate of jet velocity is approximately linear. The model predicts that the tip velocities of jets for the charges Ch1, Ch2 and the 105-mm are 6504, 4916 and $7248 \mathrm{~m} / \mathrm{s}$. respectively. In addition, the predicted rear velocities of the jet for the same charges are 1154,451 and $1887 \mathrm{~m} / \mathrm{s}$, respectively. 
Table 1. Input data to the computer program [11].

\begin{tabular}{|c|c|c|c|c|c|c|c|}
\hline $\begin{array}{c}\text { Charge } \\
\text { Design. }\end{array}$ & $\begin{array}{c}\text { Base } \\
\text { Diameter, } \\
\mathrm{D}_{\mathrm{B}} \\
{[\mathrm{mm}]}\end{array}$ & $\begin{array}{c}\text { Cone } \\
\text { Angle, } \\
2 \mathrm{a} \\
{\left[{ }^{\circ}\right]}\end{array}$ & $\begin{array}{c}\text { Cone } \\
\text { Material }\end{array}$ & $\begin{array}{c}\text { Liner } \\
\text { Density, } \\
\rho_{\mathrm{L}} \\
{\left[\mathrm{g} / \mathrm{cm}^{3}\right]}\end{array}$ & $\begin{array}{c}\text { Liner } \\
\text { Thick., } \\
\mathrm{T}_{\mathrm{L}} \\
{[\mathrm{mm}]}\end{array}$ & $\begin{array}{c}\text { Explosive } \\
\text { Type }\end{array}$ & $\begin{array}{c}\text { Expl. } \\
\text { Density, } \\
\rho_{\exp } \\
{\left[\mathrm{g} / \mathrm{cm}^{3}\right]}\end{array}$ \\
\hline Ch1 & 14.9 & 60 & Alumn. & 2.6 & 0.8 & RDX & 1.77 \\
\hline Ch2 & 14.9 & 60 & Copper & 8.9 & 0.8 & HMX & 1.89 \\
\hline $105 \mathrm{~mm}$ & 87 & 42 & Copper & 8.9 & 3.0 & Comp.B & 1.717 \\
\hline
\end{tabular}

Figure 13 plots the predicted change of normalized jet mass, $m_{j} / M_{L}$, produced from the collapsed elements of liner with relative axial position of the liner element for the charges $\mathrm{Ch} 1, \mathrm{Ch} 2$ and the $105 \mathrm{~mm}$, respectively. For each charge, the change of jet mass seems to increase exponentially with the increase of relative axial position of liner element. The predicted total jet masses from the liners of the charges $\mathrm{Ch} 1, \mathrm{Ch} 2$ and the $105 \mathrm{~mm}$ are $0.43,1.15$ and $50 \mathrm{~g}$, respectively.

The modified PER theory, as implemented into a computer program, yields a good agreement with experimental and numerical jet velocities of other investigators. In addition, the presented samples of the model predictions give the deflection angle $\delta$, the collapse angle $\beta$, the collapse velocity $V_{\text {real, }}$, the jet velocity $V_{i}$ and the jet mass $m_{i}$ as a function of each realtive axial position of liner element.

\section{CONCLUSIONS}

An analytical model has been developed to describe the jet formation process of a conical shaped cahrge. The present model is a modified version of the PER theory. New alogrithms have been applied to determine the deflection angle, the collapse angle and the collapse velocity associated with each collapsed liner element. The main equations representing the jet formation process are deived, arranged and compiled into a computer program. The input data to the program are easily determined. The predicted results of the present model are compared with experimental and numerical results of other investigators; good agreement is obtained. Moreover, represetative samples of the model predictions using small sizes and the $105 \mathrm{~mm}$ shaped charges are presented and discussed

The present model is capable of: (i) predicting the main paramters associated with the jet formation process, (ii) determining the total mass of formd jet and the time taken by each collapsed element of liner to reach the charge axis, and (iii) describing the jet formation process for other geometries of liner with a few modifications.

\section{REFERENCES}

1. Walters, P. and Zukas, J. A., "Fundamentals of Shaped Charges", Wiley Interscience Publication, John Wiley and Sons, New York, U.S.A. (1989).

2. G. Birkhoff, G., MacDpougal, D. P., Pugh, E. M. and Taylor, G. "Explosives With Lined Cavities", J. Appl. Phys., Vol. 19, pp. 563-582 (1948).

3. E. M. Pugh, E. M., Eichelberger, R. J. and Rostoker, N.," Theory of Jet Formation by Charges with Lined Conical Cavities", J. Appl. Phys., Vol. 23, No.5, pp. 532-536 (1952). 
4. Jones, D. A., "A Review of One-Dimensional Shaped Charge Theory", MRL-R-940, Defense Science and Technology Organization, Melbourne, Australia (1984).

5. Jones, D. A. "A Simple Model for Jet Formation in Shaped Charges", Propellants, Explosives, Pyrotechnics, Vol. 17, pp. $43-47$ (1992).

6. Chanteret, P. Y. "Considerations About the Analytical Modeling of Shaped Charges" Propellants, Explosives, Pyrotechnics, Vol. 18, pp. 337-344 (1993)

7. Liu Gui-Xi, "The Simplified Model for Predicting Shaped Charge Jet Parameters", Propellants, Explosives, Pyrotechnics, Vol. 20, pp. 279-282 (1995).

8. Elwani, H., "The Using Polymer Bonded Explosives to Improved Liner Shaped Charge Designs", Ph.D. Thesis, Cranfield University, U.K. (1994).

9. Hirch, E.,"Improved Gurney Formulae for Exploding Cylinders and Spheres Using Hardcore Approximations", Propellants, Explosives, Pyrotechnics, Vol. 11, pp. 81-84 (1986).

10. Battacharya, A. R., Singh, S., Kishore, P., Raychaudhuri, T. K. and Sharma, V. K., "A Simplified Approach for Development of One-Dimensional Time Dependent PC Based Code for Shaped Charge Design", $16^{\text {th }}$ Int. Symp. on Ballistics, San Francisco, CA., USA, September (1996).

11. Mohamed, S. Y.,"Factors Affecting Shaped Charge Performance", M. Sc. Thesis, M.T.C., Cairo, Egypt (2000). 

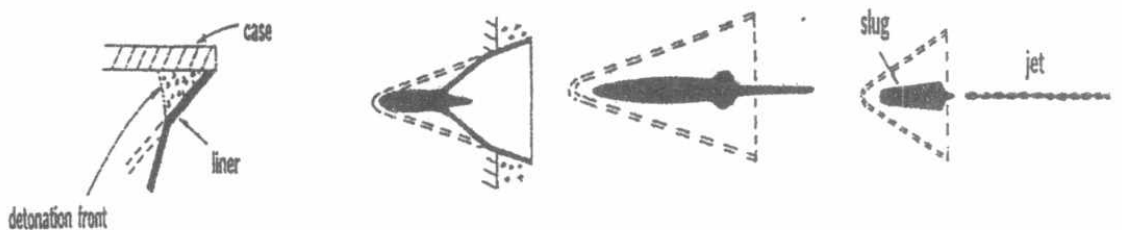

Fig. 1. Collapse process of shaped charge liner [1]

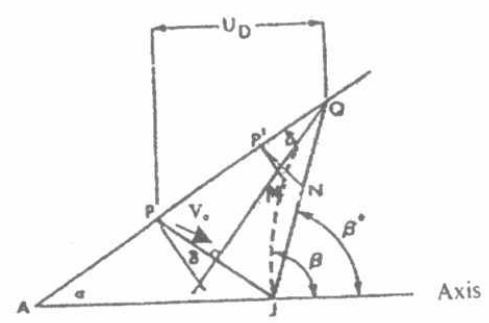

Fig. 2. Geometrical scheme of liner collapse process [1]

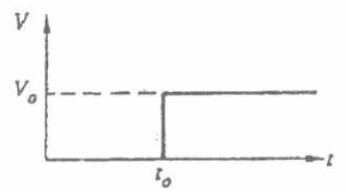

(a)

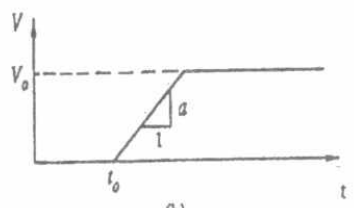

(b)

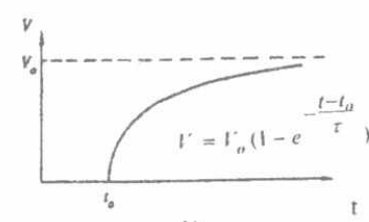

Fig. 3. Different collpase velocity-time histories of collapsed liner element $[1,8]$

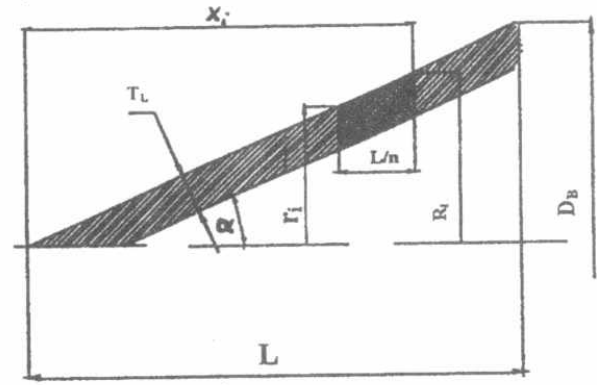

Fig. 4. Geometry of liner element 


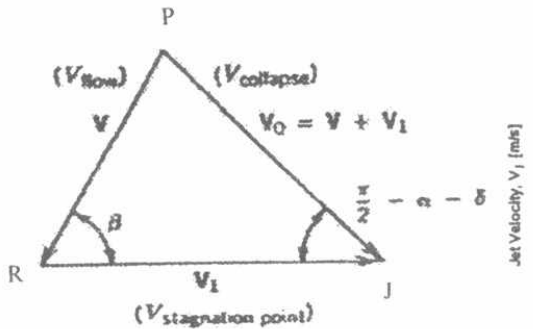

Fig. 5. Relation between real collapse velocity $\mathrm{V}_{\text {reul }}$, flow velocity $\mathrm{V}$, and collision point velocity $\mathrm{V}_{1}$

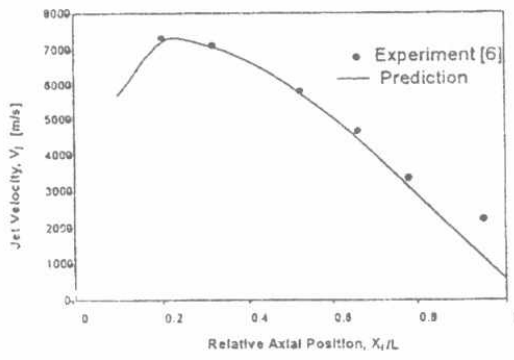

Fig. 7. The predicted change of jet velocity with relative axial position of liner for the 100 mm shaped charge, experimental results obtained by Chanteret [6].

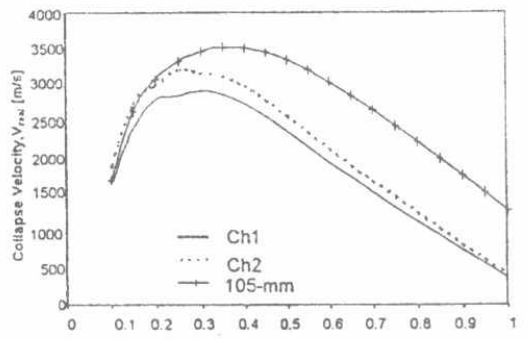

Fig. 9. The predicted change of collapse velocities with relative axial position of liner for ch1, ch2 and the 105 mm-shaped charge.

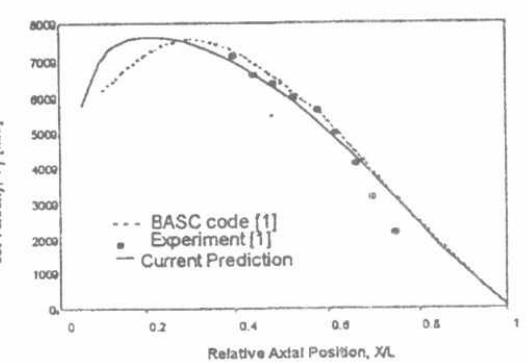

Fig. 6. The predicted change of jet velocity with relative axial position of liner for the 105 mm-shaped charge, numerical results obtained by Walters and Zukas [1], and experimental results obtained by Allison and Vitalli, see Ref. [1].

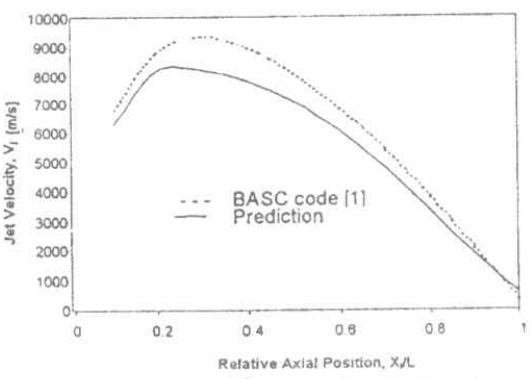

Fis. 8. The predicted change of jet velocity with relative axial position, numeric results obtained by Walter and Zukas [1].

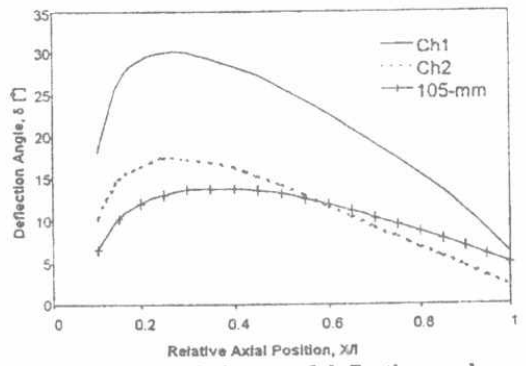

Fig. 10.The predicted change of deflection angle with relative axial position of liner for ch1, ch2 and the $105 \mathrm{~mm}$-shaped charges. 


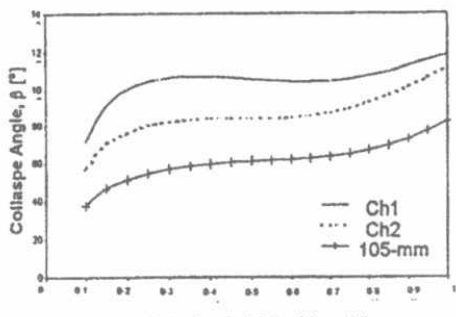

Relative Axlal Position, $\mathrm{X} / \mathrm{L}$

Fig. 11. The predicted change of collapse angle with relative axial position of liner element for Ch1, Ch2, and the $105 \mathrm{~mm}$ shaped charges

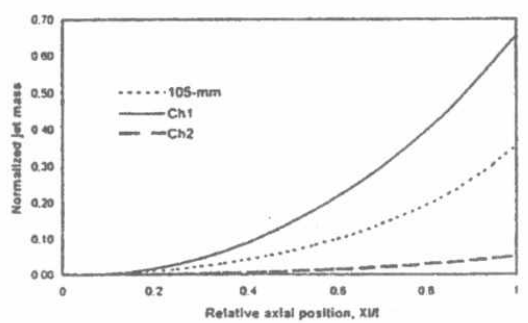

Fig. 13.The predicted change of normalized jet mass with relative axial position of liner element for $\mathrm{Ch} 1, \mathrm{Ch} 2$, and the $105 \mathrm{~mm}$ shaped charges.

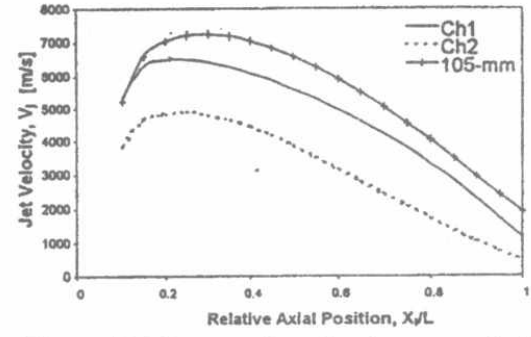

Fig. 12.The predicted change of jet velocities with relative axial position of liner for ch1, ch2 and the 105 mm-shaped charge. 